\title{
LETTER
}

\section{The angiogenic factors and their soluble receptors in sepsis: friend, foe, or both?}

\author{
Ru-Yuan Zhang, Yi-Yun Liu, Hong-Ping Qu and Yao-Qing Tang*
}

The endothelium represents an important source of, and a target for, inflammation in sepsis. Angiogenic factors and their receptors, including vascular endothelial growth factor (VEGF)/VEGF receptor and the angiopoietin/Tie2 signaling pathways, have recently received great attention in critically ill patients, including those with sepsis [1-3], because of their pivotal roles in both angiogenesis and microvascular permeability. VEGF, as the most potent proangiogenic factor, has already been identified as an important target for cancer therapy. Disassembly of an intact endothelial cell junction is necessary for the angiogenesis process. The endothelial barrier-breaking properties are indeed an important part of the VEGF role in the regulation of angiogenesis. However, a high dose or prolonged duration of VEGF may lead to excessive barrier-breaking effects. The favorable results observed with the only US Food and Drug Administration-approved anti-VEGF agent, bevacizumab, by Jeong and colleagues [4] are likely to provide potential advances for future therapeutic opportunities in sepsis.

On the contrary, VEGF shows endothelial survival signals, and suppression of VEGF signaling inhibits endothelial survival and increases apoptosis, which in turn may contribute to the development of sepsis-induced organ dysfunction [5]. Furthermore, other than an independent function on endothelial cells, VEGF plays an important role in mobilizing endothelial progenitor cells under pathologic conditions such as cancer and sepsis. While the decrease of circulating endothelial progenitor cells (cEPCs) after anti-VEGF treatment is beneficial in cancer, this may not be so in sepsis. The endothelium is a chief target of sepsisinduced events, and clinical outcome in septic patients is largely dependent on the ability to reconstitute damaged endothelium. Indeed, several studies have observed an increase in CEPC numbers in septic patients and a correlation between $\mathrm{CEPC}$ concentration and survival. The increased number of cEPCs in sepsis might therefore be

\footnotetext{
* Correspondence: yaoqing.tang@hotmail.com

Department of Critical Care Medicine, Rui Jin Hospital, Shanghai Jiao Tong University School of Medicine, 197 Rui Jin 2nd Road, Shanghai 200025, China
}

a consequence of the body's attempt to limit vascular damage by inducing endogenous endothelial repair mechanisms. The dramatic decrease of cEPCs accompanied by anti-VEGF treatment may impair endothelial repair capacity and bring unfavorable effects in sepsis.

The angiogenic factors and their soluble receptors therefore do have a complex role and seemingly play both good and bad roles during sepsis development and therapy. A certain level of VEGF is necessary for normalizing endothelial function, and abnormally high or low levels of VEGF might shift its role from endothelial protective to endothelial barrier disruptive. This view can also provide additional possible explanations for why Jeong and colleagues' study suggests that a high dose of bevacizumab may have deleterious effects in an experimental sepsis model. Future studies on the dynamic change of the angiogenic factors, their soluble receptors and cEPC counts during and after each therapy, as well as the context-dependent role of them, may provide background data for using these angiogenic factors and their soluble receptors as therapeutic targets for sepsis treatment in clinical practice.

\section{Abbreviations \\ cEPC: Circulating endothelial progenitor cell; VEGF: Vascular endothelial growth factor.}

\section{Competing interests}

The authors declare that they have no competing interests.

\section{Acknowledgements}

This work was supported by the National Natural Science Foundation of China (Grant No. 81071534).

Published: 22 August 2013

\section{References}

1. Yano K, Liaw PC, Mullington JM, Shih SC, Okada H, Bodyak N, Kang PM, Toltl L, Belikoff B, Buras J, Simms BT, Mizgerd JP, Carmeliet P, Karumanchi SA, Aird WC: Vascular endothelial growth factor is an important determinant of sepsis morbidity and mortality. J Exp Med 2006, 203:1447-1458.

2. Kumpers $P$, Lukasz A, David S, Horn R, Hafer C, Faulhaber-Walter R, Fliser D, Haller H, Kielstein JT: Excess circulating angiopoietin-2 is a strong predictor of mortality in critically ill medical patients. Crit Care 2008, 12:R147.

3. Kumpers P, van Meurs M, David S, Molema G, Bijzet J, Lukasz A, Biertz F, Haller H, Zijlstra JG: Time course of angiopoietin-2 release during experimental human endotoxemia and sepsis. Crit Care 2009, 13:R64. 
4. Jeong SJ, Han SH, Kim CO, Choi JY, Kim JM: Anti-vascular endothelial growth factor antibody attenuates inflammation and decreases mortality in an experimental model of severe sepsis. Crit Care 2013, 17:R97.

5. Jesmin S, Zaedi S, Islam AM, Sultana SN, Iwashima Y, Wada T, Yamaguchi N Hiroe M, Gando S: Time-dependent alterations of VEGF and its signaling molecules in acute lung injury in a rat model of sepsis. Inflammation 2012, 35:484-500.

doi:10.1186/1364-8535-17-446

Cite this article as: Zhang et al:: The angiogenic factors and their

soluble receptors in sepsis: friend, foe, or both?. Critical Care 2013 17:446. 